\title{
Comparison between different models of galactic tidal effects on cometary orbits
}

\author{
Marc Fouchard · Christiane Froeschlé • \\ John J. Matese • Giovanni B. Valsecchi
}

Published online: 14 November 2006

(C) Springer Science+Business Media B.V. 2006

\section{Erratum to: Celestial Mech and Dyn Astr DOI: 10.1007/s10569-005-1149-x}

Due to an unfortunate turn of events this article was published with the wrong version of Fig. 3 and with erroneous equations in the Appendices A-D therefore the correct version of Fig. 3 and the equations are published on the following pages and should be regarded by the reader as the final versions.

The online version of the original article can be found at http://dx.doi.org/10.1007/s10569-005-1149-x

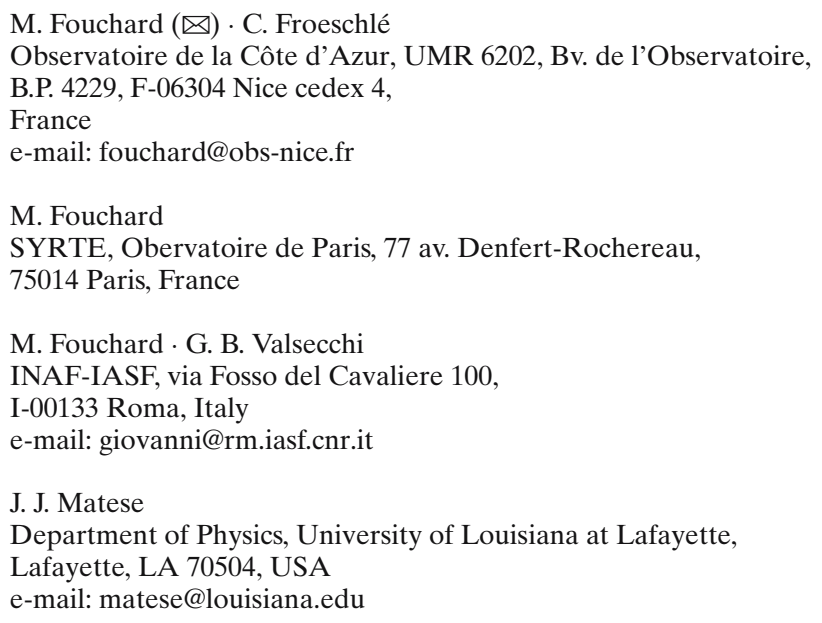


- In Appendix A, the equation for $\left\langle\frac{\mathrm{d} \Omega}{\mathrm{d} t}\right\rangle$ should read:

$$
\begin{aligned}
\left\langle\frac{\mathrm{d} \Omega}{\mathrm{d} t}\right\rangle= & -\frac{a^{2}}{4 H}\left\{( \mathcal { G } _ { 1 } - \mathcal { G } _ { 2 } ) \left[5\left(1-\frac{H^{2}}{\mu a}\right) \cos \Omega_{r} \sin \Omega_{r} \sin 2 \omega\right.\right. \\
& \left.-2 \sin ^{2} \Omega_{r} \frac{H_{z}}{H}-\left(1-\frac{H^{2}}{\mu a}\right) \frac{H_{z}}{H}(3-5 \cos 2 \omega) \sin ^{2} \Omega_{r}\right] \\
& \left.+\left(\mathcal{G}_{3}-\mathcal{G}_{2}\right) \frac{H_{z}}{H}\left[2+\left(1-\frac{H^{2}}{\mu a}\right)(3-5 \cos 2 \omega)\right]\right\} .
\end{aligned}
$$

- In Appendix B, the equation for $\left\langle\frac{\mathrm{d} H_{b}}{\mathrm{~d} t}\right\rangle$ should read:

$$
\begin{aligned}
& \left\langle\frac{\mathrm{d} H_{b}}{\mathrm{~d} t}\right\rangle=\left(\mathcal{G}_{2}-\mathcal{G}_{1}\right) \frac{a}{2 \mu \cos ^{2} b\left(H_{b}^{2} \cos ^{2} b+H_{z}^{2}\right)}\left\{-5 H_{b} \cos ^{2} b \cos l_{r}\right. \\
& \times\left(\mu a \cos ^{2} b-H_{b}^{2} \cos ^{2} b-H_{z}^{2}\right)\left(\sin l_{r} H_{z}+\cos l_{r} \sin b \cos b H_{b}\right) \\
& +\frac{1}{2} \tan b H_{z}^{2}\left(-5 \cos ^{2} l_{r} \cos ^{2} b\left(H_{b}^{2} \cos b^{2}+H_{z}^{2}\right)\right. \\
& +5\left(\sin l_{r} H_{z}+\cos l_{r} \sin b \cos b H_{b}\right)^{2} \\
& \left.+3\left(-\sin l_{r} \cos b H_{b}+\sin b \cos l_{r} H_{z}\right)^{2}-3\left(H_{b}^{2} \cos b^{2}+H_{z}^{2}\right)\right) \\
& +H_{z}\left(\sin l_{r} \cos b H_{b}-\sin b \cos l_{r} H_{z}\right) \\
& \times\left(\cos l_{r} \cos b\left(5 \mu a \cos ^{2} b-4 H_{b}^{2} \cos ^{2} b-4 H_{z}^{2}\right)\right. \\
& \left.\left.+H_{b} \sin b\left(\sin l_{r} H_{z}+\cos l_{r} \sin b \cos b H_{b}\right)\right)\right\} \\
& +\left(\mathcal{G}_{3}-\mathcal{G}_{2}\right) \frac{a}{2 \mu\left(H_{b}^{2} \cos ^{2} b+H_{z}^{2}\right)} \\
& \times\left\{-5\left(\mu a \cos ^{2} b-H_{b}^{2} \cos ^{2} b-H_{z}^{2}\right) H_{b}^{2} \sin b \cos b\right. \\
& -\tan b \frac{H_{z}^{2}}{2}\left(10 \cos ^{2} b H_{b}^{2}+8 H_{z}^{2}-8 H_{b}^{2}-8 \frac{H_{z}^{2}}{\cos ^{2} b}\right) \\
& \left.-\sin b \frac{H_{z}^{2}}{\cos b}\left(5 \mu a \cos ^{2} b-5 H_{b}^{2} \cos ^{2} b-4 H_{z}^{2}\right)\right\} \\
& +\mathcal{G}_{2} \frac{3 a H_{z}^{2} \sin b}{2 \mu \cos ^{3} b}
\end{aligned}
$$


and that for $\left\langle\frac{\mathrm{d} b}{\mathrm{~d} t}\right\rangle$ :

$$
\begin{aligned}
\left\langle\frac{\mathrm{d} b}{\mathrm{~d} t}\right\rangle= & \left(\mathcal{G}_{1}-\mathcal{G}_{2}\right) \frac{a}{2 \mu\left(\cos ^{2} b H_{b}^{2}+H_{z}^{2}\right)} \\
& \times\left\{\frac { H _ { b } } { 2 } \left(5\left(\sin l_{r} H_{z}+\cos l_{r} \sin b \cos b H_{b}\right)^{2}-5 \cos ^{2} l_{r} \cos ^{4} b H_{b}^{2}\right.\right. \\
& -5 \cos ^{2} l_{r} \cos ^{2} b H_{z}^{2}+3\left(\sin b \cos l_{r} H_{z}-\sin l_{r} \cos b H_{b}\right)^{2}-3 \cos ^{2} b H_{b}^{2} \\
& \left.\left.-3 H_{z}^{2}\right)+H_{z}\left(\tan b \cos l_{r} H_{z}-\sin l_{r} H_{b}\right)\left(\sin l_{r} H_{z}+\cos l_{r} \sin b \cos b H_{b}\right)\right\} \\
& +\left(\mathcal{G}_{3}-\mathcal{G}_{2}\right)\left(5 \cos ^{2} b-4\right) \frac{a H_{b}}{2 \mu}-\mathcal{G}_{2} \frac{3 a H_{b}}{2 \mu} .
\end{aligned}
$$

- In Appendix C, the equation for $\frac{\widehat{\mathrm{d} a}}{\mathrm{~d} t}$ should read:

$$
\begin{aligned}
\frac{\widehat{\mathrm{d} a}}{\mathrm{~d} t}= & -\frac{a^{2} H}{\mu}\left\{\left(\mathcal{G}_{2}-\mathcal{G}_{1}\right)\left(\sin 2 \Omega_{r} \cos 2 \omega \frac{H_{z}}{H}+\sin 2 \omega\left(\cos ^{2} \Omega_{r}-\sin ^{2} \Omega_{r} \frac{H_{z}^{2}}{H^{2}}\right)\right)\right. \\
& \left.+\left(\mathcal{G}_{3}-\mathcal{G}_{2}\right) \sin 2 \omega\left(1-\frac{H_{z}^{2}}{H^{2}}\right)\right\},
\end{aligned}
$$

that for $\frac{\widehat{\mathrm{d} H_{z}}}{\mathrm{~d} t}$ :

$$
\begin{aligned}
\frac{\widehat{\mathrm{d}}_{z}}{\mathrm{~d} t}= & \left(\mathcal{G}_{2}-\mathcal{G}_{1}\right)\left\{-\frac{a}{2 \mu}\left(H^{2}+3 \mu a \sqrt{1-\frac{H^{2}}{\mu a}}\right)\right. \\
& \times\left(\sin 2 \Omega_{r} \cos 2 \omega \frac{H_{z}^{2}}{H^{2}}+\sin 2 \omega \frac{H_{z}}{H}\left(\cos ^{2} \Omega_{r}-\sin ^{2} \Omega_{r} \frac{H_{z}^{2}}{H^{2}}\right)\right) \\
& \left.-\frac{5 a^{2}}{4}\left(1-\frac{H_{z}^{2}}{H^{2}}\right)\left(\cos \Omega_{r} \sin \Omega_{r}(1+\cos 2 \omega)-\sin ^{2} \Omega_{r} \sin 2 \omega \frac{H_{z}}{H}\right)\right\} \\
& +\left(\mathcal{G}_{3}-\mathcal{G}_{2}\right) \frac{a}{4 \mu}\left(-2 H^{2}+5 \mu a-6 \mu a \sqrt{1-\frac{H^{2}}{\mu a}}\right)\left(1-\frac{H_{z}^{2}}{H^{2}}\right) \frac{H_{z}}{H} \sin 2 \omega,
\end{aligned}
$$

and that for $\frac{\widehat{\mathrm{d} \omega}}{\mathrm{d} t}$ :

$$
\begin{aligned}
\frac{\widehat{\mathrm{d} \omega}}{\mathrm{d} t}= & \left(\mathcal{G}_{1}-\mathcal{G}_{2}\right)\left\{-\frac{3 a H \sqrt{\mu a}}{4 \mu \sqrt{\mu a-H^{2}}}\left(-2 \cos \Omega_{r} \sin \Omega_{r} \sin 2 \omega \frac{H_{z}}{H}\right.\right. \\
& \left.+\sin ^{2} \Omega_{r}(1-\cos 2 \omega) \frac{H_{z}^{2}}{H^{2}}+\cos ^{2} \Omega_{r}(1+\cos 2 \omega)\right) \\
& \left.+\frac{5 a^{2}}{4 H}\left(\cos \Omega_{r} \sin \Omega_{r} \sin 2 \omega \frac{H_{z}}{H}-\sin ^{2} \Omega_{r}(1-\cos 2 \omega) \frac{H_{z}^{2}}{H^{2}}\right)\right\} \\
& +\left(\mathcal{G}_{3}-\mathcal{G}_{2}\right)\left\{-\frac{3 a H \sqrt{\mu a}}{4 \mu \sqrt{\mu a-H^{2}}}\left(1-\frac{H_{z}^{2}}{H^{2}}\right)(1-\cos 2 \omega)\right. \\
& \left.+\frac{5 a^{2}}{4 H} \frac{H_{z}^{2}}{H^{2}}(1-\cos 2 \omega)\right\}-\mathcal{G}_{2} \frac{3 a H \sqrt{\mu a}}{2 \mu \sqrt{\mu a-H^{2}}} .
\end{aligned}
$$


- In Appendix D, the equation for $\frac{\widehat{\mathrm{d} a}}{\mathrm{~d} t}$ should read:

$$
\begin{aligned}
\frac{\widehat{\mathrm{d} a}}{\mathrm{~d} t}=-\frac{2 a^{2}}{\mu}\{ & \left(\mathcal{G}_{2}-\mathcal{G}_{1}\right) \cos l_{r}\left(\sin l_{r} H_{z}+\cos l_{r} \sin b \cos b H_{b}\right) \\
& \left.+\left(\mathcal{G}_{3}-\mathcal{G}_{2}\right) \sin b \cos b H_{b}\right\}
\end{aligned}
$$

Consequently, Eq. (11a) of Fouchard et al. (2005) becomes:

$$
\left\langle\frac{\mathrm{d} H_{b}}{\mathrm{~d} t}\right\rangle=\mathcal{G}_{3} \frac{a \sin b}{2 \mu \cos ^{3} b}\left\{5\left(-\mu a+H_{b}^{2}\right) \cos ^{4} b+4 H_{z}^{2}\right\}
$$

The results of Fouchard et al. (2005) are unchanged but the discussion of Section 6 can be clarified. Indeed, the force function $\mathbf{f}$ is now defined for the model $\left\langle L_{M}\right\rangle$ when $e=1$. Thus the mappings $\left[\left\langle L_{M}\right\rangle\right]_{n}$ converge toward the $\left\langle L_{M}\right\rangle$ model even when $e=1$.

Similarly, Eq. (12a) of Fouchard et al. (2005) becomes:

$$
\frac{\widehat{\mathrm{d} a}}{\mathrm{~d} t}=-\mathcal{G}_{3} \frac{a^{2} H}{\mu} \sin 2 \omega\left(1-\frac{H_{z}^{2}}{H^{2}}\right),
$$

and Eq. (13a):

$$
\frac{\widehat{\mathrm{d} a}}{\mathrm{~d} t}=-\mathcal{G}_{3} \frac{2 a^{2}}{\mu} \sin b \cos b H_{b}
$$

Consequently, Fig. 3 of Fouchard et al. (2005) should read:
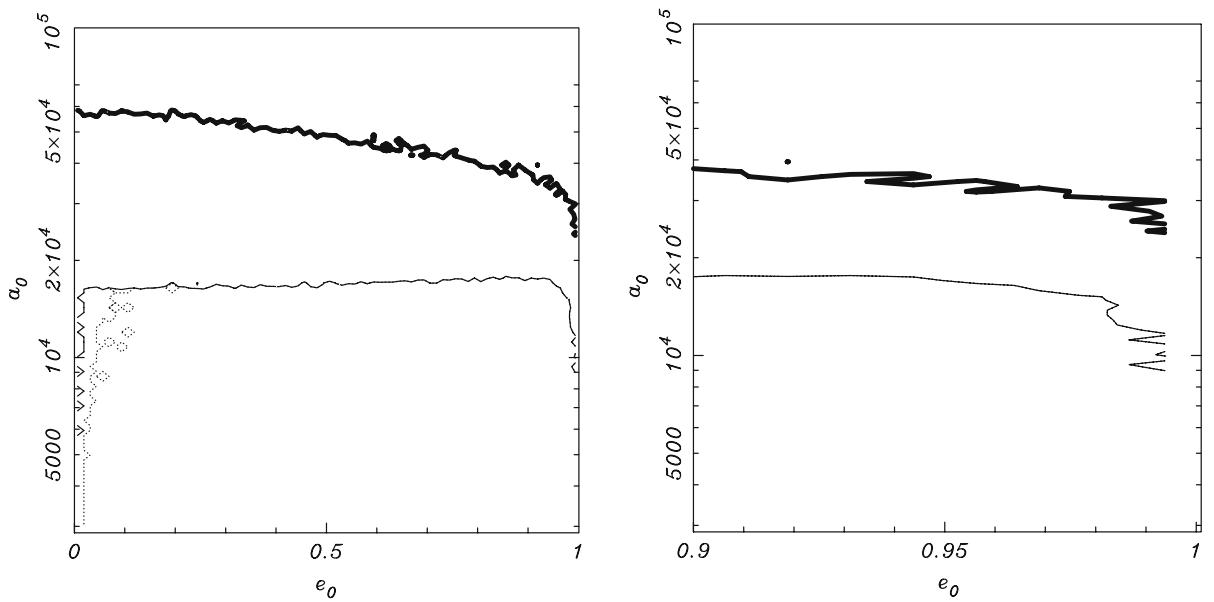

Fig. 3 Level curves in the plane $e_{0}-\log a_{0}$ when the error $E_{m}^{*}$ on the final perihelion for the 400,000 comets is equal to $1 \%$. For each level curve, one has $E_{m}^{*}>1 \%$ above the level curve, and $E_{m}^{*}<1 \%$ below the level curve. Hence, the level curve corresponds to the upper bound of the domain of reliability of the model. The level curves of the $\langle H\rangle,\langle M\rangle,\left\langle L_{H}\right\rangle$ and $\left\langle L_{M}\right\rangle$ models overlap exactly, and correspond to the thick full curve, the thin full curve to the $\widehat{L_{H}}$ model and the thin dotted curve to the $L_{M}$ model. The figure on the right is a blow-up of the figure on the left for eccentricities greater than 0.9

Anyway, these results do not change the conclusions of Fouchard et al. (2005). 